\title{
MITRAL REGURGITATION LATE AFTER MANOUGUIAN'S ANULUS ENLARGEMENT AND AORTIC VALVE REPLACEMENT
}

Kazuhito Imanaka, MD, ${ }^{\mathrm{a}}$ Shinichi Takamoto, MD, ${ }^{\mathrm{a}}$ and Akira Furuse, MD, ${ }^{\mathrm{b}}$ Tokyo, Japan

Anulus enlargement is necessary in some patients with small aortic anuli. The Manouguian procedure ${ }^{1}$ usually makes it possible to accommodate a prosthetic valve two sizes larger than the original anulus and is applicable in various situations..$^{2-5}$ Although it is a well-established

From the Department of Cardiothoracic Surgery, University of Tokyo, ${ }^{\mathrm{a}}$ and JR Tokyo General Hospital, ${ }^{\mathrm{b}}$ Tokyo, Japan.

Received for publication Nov. 4, 1997; accepted for publication Nov. 13, 1997.

Address for reprints: Kazuhito Imanaka, MD, Department of Cardiothoracic Surgery, Faculty of Medicine, University of

Tokyo, 7-3-1 Hongo, Bunkyo-Ku, Tokyo 113, Japan.

J Thorac Cardiovasc Surg 1998;115:727-9

Copyright (c) 1998 by Mosby, Inc.

$0022-5223 / 98 \$ 5.00+0 \quad \mathbf{1 2 / 5 4 / 8 7 6 9 1}$ method, its long-term results have not been well documented. Concern has been expressed about the long-term effects of this procedure on mitral valve function, because the structure of the anterior mitral leaflet and the mitral anulus are inevitably altered. Herein we report that significant mitral regurgitation (MR) often occurred late after the Manouguian procedure performed with a prosthetic patch.

Clinical summary. Four patients (one man and three women) underwent aortic valve replacement by means of the Manouguian procedure with prosthetic patches because of congenital aortic stenosis and a small anulus between 1981 and 1989. An expanded polytetrafluoroethylene (ePTFE) patch was applied in three patients, and a woven Dacron patch was used in one. Mean age at operation was 25 years. The mean follow-up period was 134.5 months. The size of the prosthetic valve was $21 \mathrm{~mm}$ in two patients, $19 \mathrm{~mm}$ in 

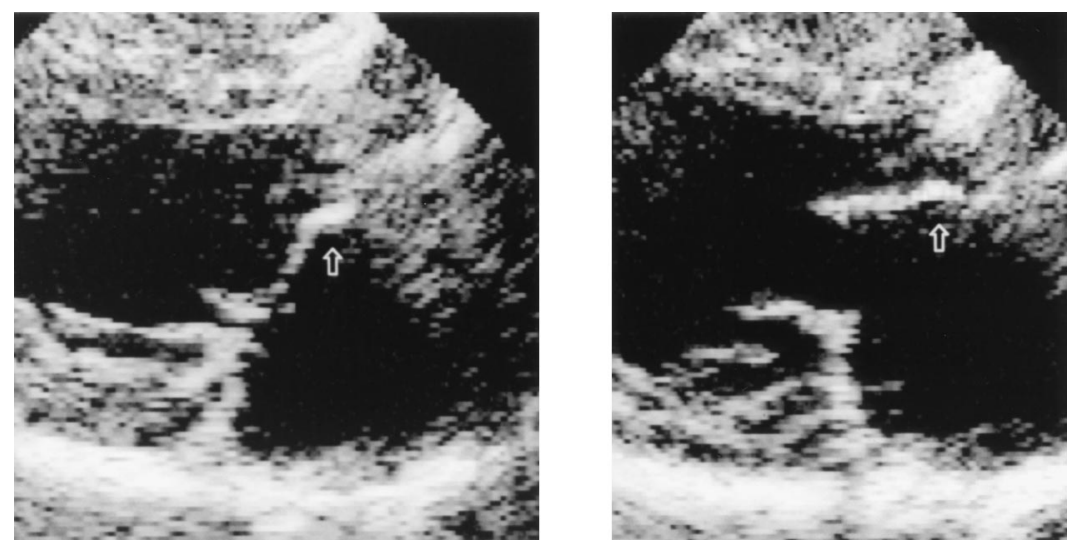

Fig. 1. Typical motion of mitral valve late after the Manouguian procedure with a prosthetic patch. Left, Systolic phase. Right, Diastolic phase. Because of the difference of mobility and stiffness between the prosthetic patch (arrow) and the native valve, the anterior leaflet appears to be bent.
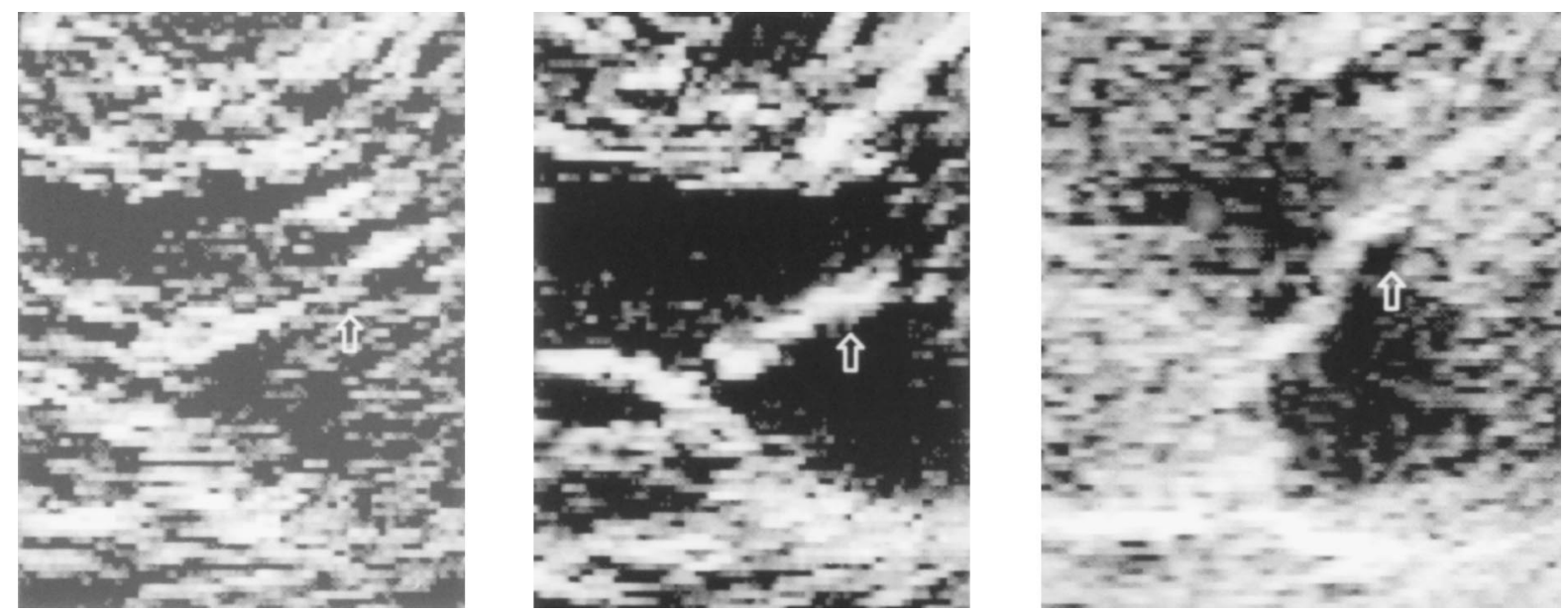

Fig. 2. Deterioration of abnormalities in the anterior mitral leaflet. Left, One year after the operation. There was no MR. Middle, Six years later. Mild MR was observed. Right, Twelve years later. Moderate degree of MR was present. Arrow, Prosthetic patch.

one, and $17 \mathrm{~mm}$ in one. Mitral prolapse and MR developed late after the operation in all four patients. In three of four patients who had been followed up longer than 10 years, mitral prolapse was marked and the grade of MR was greater than moderate. One patient whose follow-up period was 7 years had mild prolapse and mild MR. The anterior leaflet prolapsed and regurgitant flow was oriented toward the posterior wall of the left atrium. Mobility of the basal portion of the anterior mitral leaflet was poor. This portion was highly echogenic and thought to be prosthetic material. On the other hand, the marginal portion of the anterior leaflet was fully mobile but prolapsed. The echogenicity of this portion was normal or only slightly enhanced. Inasmuch as mobility differed markedly between the native mitral valve and the prosthetic patch, the anterior leaflet appeared to have a joint with a bend at the junction (Fig. 1). However, the abnormal motion, prolapse, and MR were absent in the early postoperative period. Serial echocardiographic studies in two patients with an ePTFE patch revealed that the mitral valve had initially appeared almost normal. Abnormal findings in the mitral valve and MR became apparent about 5 years later and then deterioration increased (Fig. 2). Such an abnormal motion or significant MR were not observed in four patients in whom autologous pericardium had been used as a patch, although mild mitral prolapse was commonly seen in these patients as well.

Comments. We found that significant MR resulting from mitral prolapse was common in patients who had undergone the Manouguian procedure with a prosthetic patch. It is clear that poorly flexible, firm material is unsuitable for the patch, because it forms a part of the mitral valve. However, significant MR also occurred in patients in whom ePTFE, an originally soft material, was used. The fact that the abnormalities in the mitral valve developed at a late postoperative stage and were progressive strongly suggests that the cause of MR in these patients was patch degeneration rather than an 
inappropriate incision. ${ }^{2}$ We actually encountered strikingly severe degeneration of ePTFE at a reoperation 7 years after the Manouguian procedure. The patch was too stiff to function as part of the heart valve, although prolapse and MR were mild in this case. Therefore it appears better not to use synthetic prosthetic patches that may degenerate later on. In this regard, bioprosthetic material may also not be a good choice for the patch. Even allografts are not free from degeneration. In our experience, patients with an autologous pericardial patch had better results. Abnormal motion of the mitral valve or significant MR was not present in any of them. However, shrinkage and sclerosing change of the autologous pericardium are also well known. At present, the ideal patch material for the Manouguian procedure does not seem to exist. In addition, acceptable morphologic alteration of the mitral anulus and the anterior leaflet in this procedure is yet unclear, especially in pediatric patients. Therefore every patient undergoing the Manouguian procedure needs careful follow-up.

\section{REFERENCES}

1. Manouguian S, Seybold-Epting W. Patch enlargement of the aortic valve ring by extending the aortic incision into the anterior mitral leaflet. J Thorac Cardiovasc Surg 1979;78:40212 .

2. Kawachi Y, Tominaga R, Tokunaga K. Eleven-year follow-up study of aortic or aortic-mitral anulus-enlarging procedure by Manouguian's technique. J Thorac Cardiovasc Surg 1992;104: 1259-63.

3. Okabe H, Asano K, Mizuno A, et al. Clinical and anatomical evaluation of Manouguian's procedure. J Jpn Assoc Thorac Surg 1986;34:1884-91.

4. Manouguian S, Abu-Aishah N, Neitzel J. Patch enlargement of the aortic and mitral valve rings with aortic and mitral double valve replacement. J Thorac Cardiovasc Surg 1979;78: 394-401.

5. Furuse A, Mizuno A, Asano K. Aortoatrioplasty with double valve replacement for infective endocarditis. J Cardiovasc Surg 1984;25:462-6. 